\title{
The response of hot wires in high Reynolds-number turbulent pipe flow
}

\author{
J D Li ${ }^{1}$, B J McKeon, W Jiang ${ }^{2}$, J F Morrison ${ }^{3}$ and A J Smits \\ Department of Mechanical and Aerospace Engineering, Princeton University, Princeton, \\ NJ 08544-0710, USA
}

Received 2 October 2003

Published 26 March 2004

Online at stacks.iop.org/MST/15/789 (DOI: 10.1088/0957-0233/15/5/003)

\begin{abstract}
Issues concerning the accuracy of hot-wire measurements in turbulent pipe flow are addressed for pipe Reynolds numbers up to $6 \times 10^{6}$ and hot-wire Reynolds numbers up to $R e_{w} \approx 250$. These include the optimization of spatial and temporal resolution and the associated feature of signal-to-noise ratio. Very high wire Reynolds numbers enable the use of wires with reduced length-to-diameter ratios compared to those typical of atmospheric conditions owing to increased wire Nusselt numbers. Simulation of the steady-state heat balance for the wire and the unetched portion of wire are used to assess static end-conduction effects: they are used to calculate wire Biot numbers, $\sqrt{c_{0}} l$, and fractional end-conduction losses, $\sigma$, which confirm the 'conduction-only' theory described by Corrsin. They show that, at $R e_{w} \approx 250$, the wire length-to-diameter ratio can be reduced to about 50, while keeping $\sqrt{c_{0}} l>3$ and $\sigma<7 \%$ in common with accepted limits at $R e_{w} \approx 3$. It is shown that these limits depend additionally on the choice of wire material and the length of unetched wire. The dynamic effects of end-cooling are also assessed using the conduction-only theory.
\end{abstract}

Keywords: fluid flow velocity, thermal anemometry, turbulence diagnostics

\section{Introduction}

Despite comprehensive studies on the effects of fluid flow around hot wires (Brunn 1995, Collis and Williams 1959, Corrsin 1963, Fingerson and Freymuth 1983, Hinze 1975, Perry 1982), attention has focused on measurements at quite low Reynolds numbers, often at atmospheric pressure. In detailed studies using normal hot wires in fully-developed pipe flow at pressures up to $120 \mathrm{~atm}$ (Morrison et al 2002a, 2002b, 2004), it has become apparent that some difficulties associated with hot-wire velocity measurement are particular to high Reynolds numbers. The most obvious of these are temporal resolution related to the anemometer frequency response, and spatial resolution related to wire length and the detailed behaviour of the hot wire itself.

1 Permanent address: School of the Built Environment, Victoria University of Technology, PO Box 14428, MCMC, Melbourne, Australia.

2 Permanent address: CARDC, PO Box 211 Mianyang, Sichuan 621000, People's Republic of China.

3 Permanent address: Department of Aeronautics, Imperial College, London SW7 2AZ, UK.
Typically, at atmospheric conditions, the Reynolds number based on wire diameter, $R e_{w}$, is of order 10 , leading to a corresponding wire Nusselt number, $N u_{w}$ of about 2. By comparison, at $120 \mathrm{~atm}, R e_{w} \approx 250$ and $N u_{w} \approx 10$. Note that a 25-fold increase in $R e_{w}$ leads to an approximate 5-fold increase in $N u_{w}$ because a typical forced convection law, for example that of Fand and Keswani (1972), is given by

$$
N u_{w}=\left(0.35+0.56 \operatorname{Re}_{w}^{0.52}\right) \operatorname{Pr}^{0.3}
$$

for $0.1<R e_{w}<10000$, where $\operatorname{Pr}$ is the Prandtl number of the fluid which changes only slightly with pressure. Modelling a hot-wire system using a third-order transfer function (with a quadratic pole approximation-see Freymuth (1977), Perry (1982)), it can be shown that the effect of increasing the mean cooling velocity on the wire decreases the damping so that the system tends to become unstable. Owing to the form of the cooling law, equation (1.1), the same tendency occurs at high Reynolds numbers, even if the mean velocity is low. To some extent, the situation can be improved by careful tuning of the variable bridge inductance. Ultimately however, the amplifier gain needs to be reduced to maintain stability, so reducing the frequency response. With newer anemometer 
designs, improvements in frequency response are likely and a higher order transfer function may be needed to model these improved systems. Nevertheless, the optimal thirdorder frequency response to a square-wave input (Freymuth 1977), as measured by the cut-off frequency $f_{c}$, becomes more important at large $R e_{w}$. Here,

$$
f_{c}=\frac{1}{1.3 \tau}
$$

where $\tau$ is the time between the start of the pulse, and the first zero crossing.

For a hot-wire length, $2 l$, the Fourier transform of a 'tophat' response function in space yields

$$
S(k)=\left[\frac{\sin k l}{k l}\right]^{2},
$$

where $k$ is the wavenumber. Equation (1.3) has zeros at $k l=\pi$ so that an estimate of the expected spatial resolution is given by $k=\pi / l$. In a facility in which a high Reynolds number is obtained by a means other than a high velocity (Zagarola 1996, Zagarola and Smits 1998, McKeon et al 2004, Morrison et al 2002a, 2002b, 2004), any limitation to the resolution of the data is more likely to be determined by the spatial resolution of the hot wire than by the frequency response of the bridge. In the present case, use of high density to achieve a high Reynolds number permits the use of a low mean velocity so that wavenumber resolution is compromised less by a poor anemometer frequency response than it would be at higher mean velocities. Yet the question of spatial resolution remains.

It is therefore important to determine whether or not a reduction in length-to-diameter ratio, $2 l / d$, is possible. In principle, the increase in $N u_{w}$ at very high Reynolds number leads to an increase in forced convection from the wire, $\psi$, relative to conduction to the prongs, $K$, as suggested by Corrsin (1963) and Freymuth (1979). To ensure that the temperature distribution along the wire is as uniform as possible, the minimum recommended value for $2 l / d$ is often quoted to be about 200 for a cooling velocity of order $10 \mathrm{~m} \mathrm{~s}^{-1}$ atmospheric pressure. It is obvious therefore that the thermal conductivity of the hot wire and its support should be as low as possible. For turbulence measurements at high Reynolds numbers, hot wires of small diameters $(d=1 \mu \mathrm{m})$ are preferable, so that the length is reduced also. However, present experience suggests that a practical minimum diameter is about $2.5 \mu \mathrm{m}$ to provide a sufficiently robust sensor.

Hot-wire temperature distributions have previously been examined in detail by Brunn (1995), Champagne et al (1967), Corrsin (1963), Fingerson and Freymuth (1983), Freymuth (1979), Hinze (1975) and Perry (1982). Typically, these analyses assume that the temperature of the end of the wire is the same as that of the ambient fluid. The exceptions are those of Champagne et al (1967), in which experimental results for the temperature at the end of the hot wire are used, and those in Perry (1982), where it is assumed that the temperature distribution along the hot-wire support (here, the 'stub' of plated or unetched wire) is a linear function of axial distance. Such an assumption is valid only when the stub is sufficiently large so that convection from, and heat generation within the stub are negligible and only the effects of stub conduction are modelled (Corrsin 1963, Fingerson and Freymuth 1983,
Freymuth 1979). The effect of the hot-wire support on the heat balance of the hot wire has not been studied previously without invoking these simplifying assumptions. Moreover, in all these analyses, the effect of Reynolds number has not been discussed.

A measure of the relative importance of static end-cooling effects may be defined by the heat transfer ratio

$$
\sigma=\frac{K}{K+\psi}
$$

see Freymuth (1979). An equivalent definition is also provided by the use of a Biot number, a measure of the convective transfer coefficient of the fluid relative to the thermal conductivity of the wire (and defined in the following section). Corrsin (1963) provides full details. In the present paper, the heat-balance equation for a hot wire with a stub under steady-state conditions is solved numerically. This includes the heat generation within the stub and forced convection from it. Although generally a small effect, stub convection can become significant when $2 l / d$ is large and the stub temperature is significantly higher than the ambient temperature. Moreover, when the stub is long so that its resistance becomes significant, heat generation within the stub can also become important. The purpose of the present analysis is to identify how these effects change with Reynolds number, geometry and hot-wire material properties. At small $2 l / d$ (the focus of the present study for hot-wire measurements at high Reynolds number), stub-conduction effects become important and identification of an acceptable maximum value of $\sigma$ (or an acceptable minimum Biot number) provides an allowable minimum of $2 l / d$ to be specified for a given $R e_{w}$, stub geometry, and thermal conductivities of the stub and wire.

In the light of this analysis, the related issue of frequency response is examined experimentally. Without dynamic calibration (impractical in this case), any differences between a dynamic and static calibration are especially important at high Reynolds numbers if length-to-diameter ratios are reduced. Freymuth (1979) used a linear, 'quasi-static' analysis - see also Hinze (1975) — to consider the dynamic effects of end losses. Corrsin (1963) explains these effects by considering the non-dimensional 'relaxation' times for the physical processes involved. A conduction time constant given by $(2 l)^{2} / \wp$, where $\wp$ is the thermal diffusivity, is typically several orders of magnitude larger than one associated with the convection of small scales past the hot wire, $2 l / U$, where $U$ is an appropriate convection velocity. Therefore, even if the flow is isothermal (as in the present case), the Biot number depends on time as well as axial position so that some parts of the wire respond more slowly to velocity changes than others. Similarly, heat generation within the wire also depends on time and axial position. This imbalance leads to heat waves of conduction along the wire, which Hinze (1975) explains as a phase shift between velocity fluctuations and resistance fluctuations. Heat waves may be minimized by using a high overheat ratio.

A dynamic analysis of the heat-balance equation-see, for instance, Hinze (1975), Perry (1982)-leads to intractable solutions without simplifying assumptions being made. If the time constant of the wire is taken to be negligible, the lowfrequency effects of end-cooling may be isolated (Perry et al 1979). Corrsin (1963), Fingerson and Freymuth (1983) 
and Freymuth (1979) show that the one-dimensional heatconduction equation for temperature along the wire has an exponential solution so that heat waves are attenuated above the circular frequency given by

$$
\omega_{l} \approx 40 \frac{\wp}{(2 l)^{2}} .
$$

For $\omega \ll \omega_{l}$, the effect of heat waves is small since the time constant associated with conduction is of the same order as those associated with the fluid motion. Therefore at low frequencies, the wire temperature distribution will be close to the mean. However, for $\omega>\omega_{l}$, heat waves are attenuated so that wire temperatures are reduced near the middle of the wire, but increased near its ends, producing an attenuated response with a reduced average Biot number (Fingerson and Freymuth 1983). Freymuth (1979) defines the ratio

$$
\sigma^{\prime}=\frac{\mathrm{d} K}{\mathrm{~d} K+\mathrm{d} \psi},
$$

in which $\mathrm{d} K$ and $\mathrm{d} \psi$ represent, respectively, the rates of change of $K$ and $\psi$ with Biot number. These quantities are straightforwardly defined by his analysis of end losses by conduction. Equations (1.5) and (1.6), which ignore the feedback of the electronic circuit (constant temperature mode), are used to examine dynamic end-cooling effects in the context of the present measurements. Using a time-dependent solution of the heat-balance equation and including a model of the electronic feedback, Morris and Foss (2003) have recently confirmed Freymuth's (1979) analytical quasi-static solution for end-conduction losses. They show that the effect of heat waves becomes apparent at a frequency close to that given by equation (1.5) and confirm an asymptotic value of $\sigma^{\prime}=0.07$ for the same wire and conditions, $R e_{w} \approx 3$.

A further practical limitation to anemometer output frequency, $f$, response has been documented in Freymuth and Fingerson (1997) and Saddoughi and Veeravalli (1996): output noise from conventional analogue bridges leads to velocity power spectra in which the spectral density increases in proportion to $f^{2}$ (a 'ramp') beginning at $2-5 \mathrm{kHz}$. The effect has been analysed by Freymuth (1968), and is intrinsic to the way in which analogue anemometers work: as the frequency increases, the thermal response of a hot wire decreases and the gain, and therefore noise amplification, of the compensating circuit must increase. Nevertheless, this is potentially a very serious condition because it can lead to quite far-reaching, yet erroneous, conclusions in the interpretation of data at high Reynolds numbers. For example, it is likely that the limitations set by spatial resolution will become apparent in the inertial subrange, and a physical roll-off below the $-5 / 3$ slope might well be compensated by the effects of the $f^{2}$. ramp leading to a false conclusion concerning the extent of the inertial subrange. The effect may be minimized by using a high overheat ratio, as thin a wire as possible (to reduce thermal inertia) and wire material with a high temperature coefficient of resistivity (Freymuth and Fingerson 1997). It is also possible, but has not yet been demonstrated, that newer designs of analogue anemometers have a better signal-to-noise ratio.

In what follows, we assess the static effects of endconduction losses by a numerical solution which is used to consider the separate effects of Reynolds number, stub length

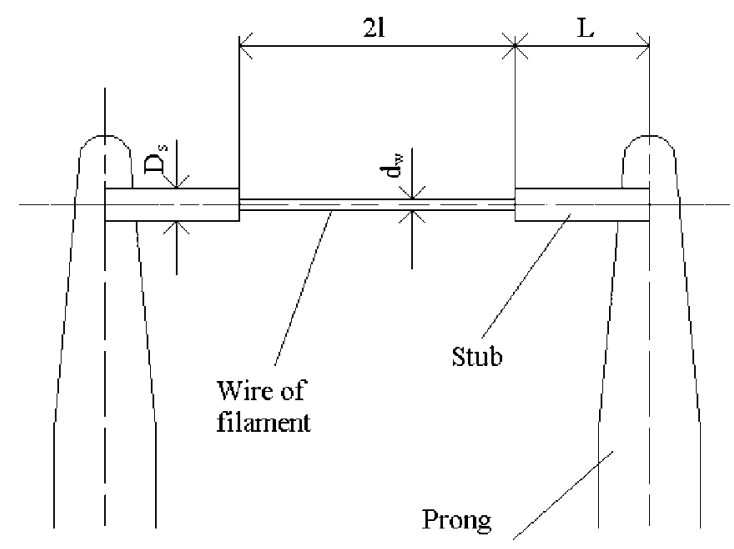

Figure 1. Hot-wire geometry (not to scale).

and wire material for a range of $2 l / d$. We also use Freymuth's analysis for assessing the dynamic response of the hot wires used with $2 l / d \approx 100$ up to $R e_{w} \approx 250$. Earlier calculations of end losses have been presented by Li (2001).

\section{Numerical results}

\subsection{Heat transfer analysis of the hot wire}

Figure 1 shows a sketch of the hot-wire geometry. The distance along the wire (measured from its centre, about which we take the wire and the flow to be symmetrical) is given by $\chi=x / l$. Using subscripts $x$ and $a$ to denote wire/stub and ambient conditions respectively, the steady-state balance of the generation, convection and conduction of heat along the wire satisfies the following equation:

$$
\frac{\mathrm{d}^{2} \theta}{\mathrm{d} \chi^{2}}-(p-q) \theta+Z=0
$$

where the temperature ratio is given by

$$
\theta=\frac{\theta_{x}-\theta_{a}}{\theta_{a}}
$$

and the non-dimensional convection is

$$
p=4 N u_{w} \frac{k_{f}}{k_{x}}\left(\frac{l}{d}\right)^{2} .
$$

For a detailed derivation, see Perry (1982). Writing $I$ for the wire current, $R_{x}$, for the wire resistance per unit length, and $\alpha$, for the linear temperature coefficient of electrical resistance,

$$
R_{x}=R_{a}\left[1+\alpha\left(\theta_{x}-\theta_{a}\right)\right],
$$

the heat generated is given by two terms,

$$
q=\frac{4 I^{2} R_{x} \alpha}{\pi k_{x}}\left(\frac{l}{d}\right)^{2},
$$

and

$$
Z=\frac{4 I^{2} R_{x}}{\pi k_{x} \theta_{a}}\left(\frac{l}{d}\right)^{2} .
$$

Here, $k_{x}$ and $k_{f}$ are the thermal conductivities of the wire (or stub) and fluid, respectively. Note that, in general, the diameter, $d$, is a function of axial distance: along the wire, it is constant and the stub diameter, $D_{s}$, is also. For simplicity, the change in diameter has been assumed to be a step function 
Table 1. Mechanical, thermal and electrical properties for platinum $(\mathrm{Pt})$, platinum/rhodium $(\mathrm{Pt} / \mathrm{Rh})$, tungsten $(\mathrm{W})$ and titanium/nickel $(\mathrm{Ti} / \mathrm{Ni})$.

\begin{tabular}{|c|c|c|c|c|}
\hline Material & $\mathrm{Pt}$ & $\begin{array}{l}\mathrm{Pt} / \mathrm{Rh} \\
(90 / 10 \%)\end{array}$ & W & $\mathrm{Ti} / \mathrm{Ni}$ \\
\hline Tensile strength (MPa) & 620 & 700 & 750 & 560 \\
\hline $\begin{array}{l}\text { Specific heat capacity, } \\
C_{w}\left(\mathrm{~kJ} \mathrm{~kg}^{-1} \mathrm{~K}^{-1}\right)\end{array}$ & 0.13 & 0.15 & 0.14 & 0.32 \\
\hline $\begin{array}{l}\text { Thermal conductivity, } \\
k_{w}\left(\mathrm{~W} \mathrm{~m}^{-1} \mathrm{~K}^{-1}\right)\end{array}$ & 69 & 38 & 170 & 10 \\
\hline Melting point $(\mathrm{K})$ & 2042 & 2103 & 3643 & 1513 \\
\hline Resistivity $(\Omega \mathrm{m})$ & $1.9 \times 10^{-7}$ & $1.9 \times 10^{-7}$ & $5.7 \times 10^{-8}$ & $8.2 \times 10^{-7}$ \\
\hline $\begin{array}{l}\text { Temperature coefficient of } \\
\text { resistivity, } \alpha\left(\mathrm{K}^{-1}\right)\end{array}$ & 0.0035 & 0.0019 & 0.0052 & 0.0038 \\
\hline
\end{tabular}

and the stub is cylindrical. It is assumed that the temperature at the junction between the stub and the supporting prong is that of the ambient fluid because the prong is much bulkier in volume than the stub. Thus the temperature distribution along the wire satisfies the following boundary conditions:

$$
\begin{gathered}
\theta=0 ; \quad \chi=1+\frac{L}{l} \\
\frac{\mathrm{d} \theta}{\mathrm{d} \chi}=0 ; \quad \chi=0,
\end{gathered}
$$

where $L$ is the stub length. For $\chi \leqslant 1.0, k_{x}=k_{w}$, the thermal conductivity of the wire material. For $\chi>1.0, k_{x}=k_{s}$, the thermal conductivity of the stub. Equation (2.1) together with the boundary conditions of equations (2.7) and (2.8) is solved numerically. Five thousand uniformly-spaced grid points were used between $\chi=0$ and $\chi=1+L / l$.

For constant-temperature operation of the hot wire, the electrical current, $I$, is specified so that the overheat ratio, $a$, satisfies

$$
\alpha \theta_{a} \int_{0}^{1} \theta \mathrm{d} \chi=a=\alpha\left(\theta_{w}-\theta_{a}\right),
$$

where $\theta_{w}$ is the average wire temperature,

$$
\theta_{w}=\int_{0}^{1} \theta_{x} \mathrm{~d} \chi
$$

Owing to the integral form of equation (2.9), it is solved using an iterative procedure. The solution uses the Nusselt number given by equation (1.1). It should be noted that, while the increase of electrical resistance in the stub due to its temperature being above ambient is taken into account in the solution of equation (2.1), it is neglected in estimating the overheat ratio, equation (2.9), because the overall resistance of the stub is much smaller than that of the wire. In the calculations, we take values for air under laboratory conditions, $\operatorname{Pr}=0.7, \theta_{a}=293 \mathrm{~K}$ and $k_{f}=0.0257 \mathrm{~W} \mathrm{~m}^{-1}$ $\mathrm{K}^{-1}$. Solutions are presented for $a=0.82, D_{s} / d=5$. Results at $a=0.4$ and $D_{s} / d=10$ were also calculated, but these parameters have only a small influence on a minimum lengthto-diameter ratio. We also take the thermal conductivity of the stub to be that of silver, $k_{s}=429 \mathrm{~W} \mathrm{~m}^{-1} \mathrm{~K}^{-1}$, corresponding to Wollaston wires that use silver plating. However, comparison with the wire thermal conductivities given in table 1 shows that this stub conductivity, being several times that of the wire, is unsuitable from this point of view. In our calculations, nevertheless, it serves the very useful purpose of highlighting the relative effects of the stubs at small and large $2 l / d$.

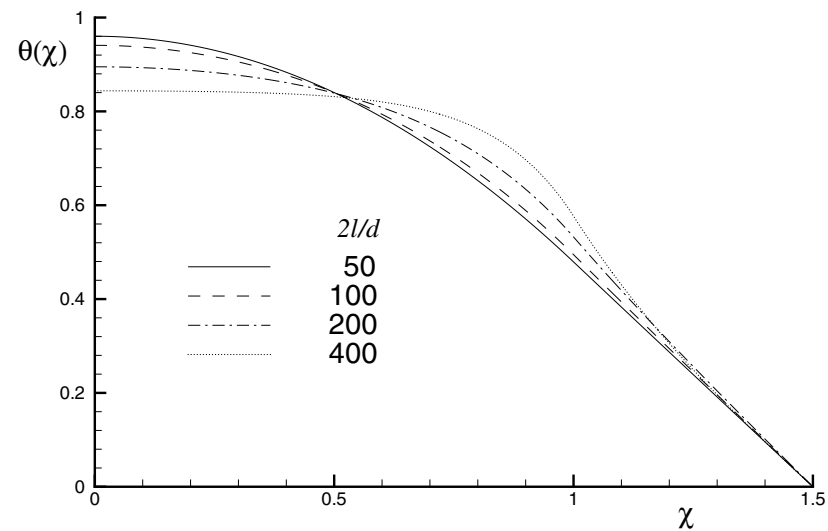

Figure 2. Temperature distribution, $\theta(\chi)$, along the wire and stub: Pt wire $(d=5 \mu \mathrm{m}), L / l=0.5, \operatorname{Re}_{w}=3.2, a=0.82$.

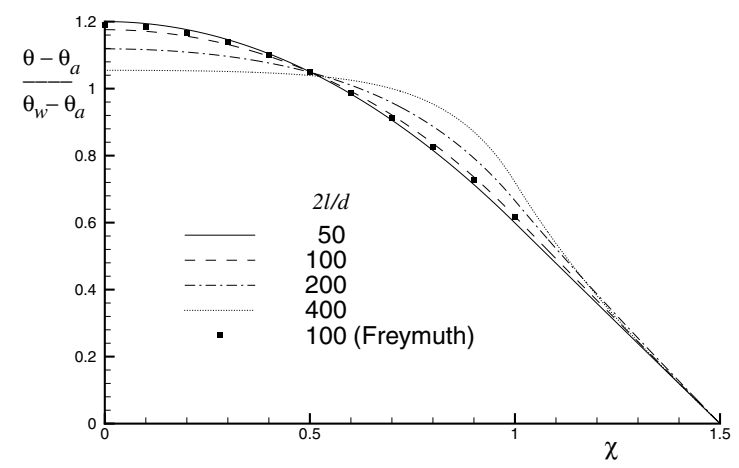

Figure 3. Temperature distribution, $\left(\theta_{x}-\theta_{a}\right) /\left(\theta_{w}-\theta_{a}\right)$, along the wire and stub: same conditions as in figure 2 .

\subsection{The effect of hot-wire support}

Figure 2 shows solutions for the temperature distribution, $\theta(\chi)$, along a $5 \mu \mathrm{m}$ platinum $(\mathrm{Pt})$ wire with $L / l=$ $0.5, D_{s} / d=5, R e_{w}=3.2$ (for an air velocity of $10 \mathrm{~m} \mathrm{~s}^{-1}$ at atmospheric conditions) and $a=0.82$ at four values of $2 l / d=50,100,200$ and 400 . It can be seen that, as $2 l / d$ increases, the temperature distribution along the wire becomes more uniform and that therefore $\sigma$ is reduced. For comparison, figure 3 shows the same data plotted as $\left(\theta_{x}-\theta_{a}\right) /\left(\theta_{w}-\theta_{a}\right)$ versus $\chi$. Table 2 shows the corresponding Biot numbers, $\sqrt{c_{0}} l$, defined by

$$
\sqrt{c_{0}} l=\frac{2 l / d\left[\left(k_{a} / k_{w}\right) N u_{w}\right]^{1 / 2}}{\left[1+\alpha\left(\theta_{w}-\theta_{a}\right)+\alpha\left(\theta_{w}-\theta_{s}\right) /(\xi-1)\right]^{1 / 2}}
$$


The response of hot wires at high Reynolds numbers

Table 2. Freymuth model estimates of $\sigma$ and $\sigma^{\prime}$ for data of figures 2 and 3 .

\begin{tabular}{rrlll}
\hline $2 l / d$ & $\sqrt{c_{0} l}$ & $\xi$ & $\sigma$ & $\sigma^{\prime}$ \\
\hline 50 & $\sim 0.01$ & 1.00 & 0.5570 & 0.5550 \\
100 & 0.46 & 1.07 & 0.4730 & 0.0464 \\
200 & 2.88 & 2.90 & 0.0622 & 0.0182 \\
400 & 6.17 & 6.17 & 0.0276 & 0.0115 \\
\hline
\end{tabular}

where $\xi=\sqrt{c_{0}} l \operatorname{coth} \sqrt{c_{0}} l$ and $\theta_{s}$ is the temperature of the stub (Freymuth 1979, Fingerson and Freymuth 1983).

At small $2 l / d$, a non-uniform temperature distribution along the wire arises as the result of conduction to the stubs. Consequently, the stub temperature decreases and so the effects of heat generation within the stub and convection from it are diminished. Conversely, at large $2 l / d$, conduction to the prongs is small, and the stub temperature rises leading to increased heat generation within the stub and convection from it. Figure 2 shows that the temperature distribution for large $2 l / d$ near the wire-stub junction, $\chi \approx 1.0$, is nonlinear because of these effects, which are accentuated by the large value of conductivity chosen for the stub. Previous analyses, such as that of Perry (1982), have assumed a linear temperature distribution at the wire-stub junction, so neglecting the small effects of generation within the stub and convection from it. The point of inflection in the temperature distribution for large $2 l / d$ is indicative of these processes. The curvature of the temperature profiles is further accentuated by the assumption of a step-change in wire-stub diameter. However, it is more usual that etching, the standard method for producing hot wires, does not always produce such a step-change.

Figure 3 may usefully be compared with the analytical solution for end-conduction effects given by Corrsin (1963), Freymuth (1979) and others. Such a comparison has greater validity when the effects of heat generation within the stub and convection from it are small relative to the conduction losses, that is, when $2 l / d$ is small. Figure 3 also shows the analytical solution of Freymuth (1979) for $2 l / d=100$ and taking $\theta_{s}=\theta_{x}(1)$. For $\theta_{a}=20{ }^{\circ} \mathrm{C}$, the temperature at the junction is about $160{ }^{\circ} \mathrm{C}$ at $2 l / d=50$ and $190{ }^{\circ} \mathrm{C}$ at $2 l / d=400$. These temperatures lead to wire-stub junction values of $\left(\theta_{x}-\theta_{a}\right) /\left(\theta_{w}-\theta_{a}\right) \approx 0.6$, significantly higher than is expected by the analytical solutions for end-conduction effects because of the long stub lengths used in the present calculations. When $L / l$ is reduced to 0.1 , simulation shows the temperature at the end of the wire to be about $60{ }^{\circ} \mathrm{C}$, $\left(\theta_{x}-\theta_{a}\right) /\left(\theta_{w}-\theta_{a}\right) \approx 0.2$. This value of $L / l$ is close to that used by Champagne et al (1967), where the measured temperature at the end of the hot wire was also about $60{ }^{\circ} \mathrm{C}$ for similar wires. This shows that the length of the stub support for the hot wire has potentially a large effect on the uniformity of the wire temperature distribution.

Figure 4 shows the relative heat loss due to conduction, $\sigma$, for $L / l=0.25$ and 1.0 with $50 \leqslant 2 l / d \leqslant 400$. The wire material and the other operating conditions are the same as those in figures 2 and 3 . Here the conduction, $K$, is given by

$$
K=-\left.\frac{\pi d^{2}}{4 l} k_{w} \theta_{a} \frac{\mathrm{d} \theta}{\mathrm{d} \chi}\right|_{\chi=1},
$$

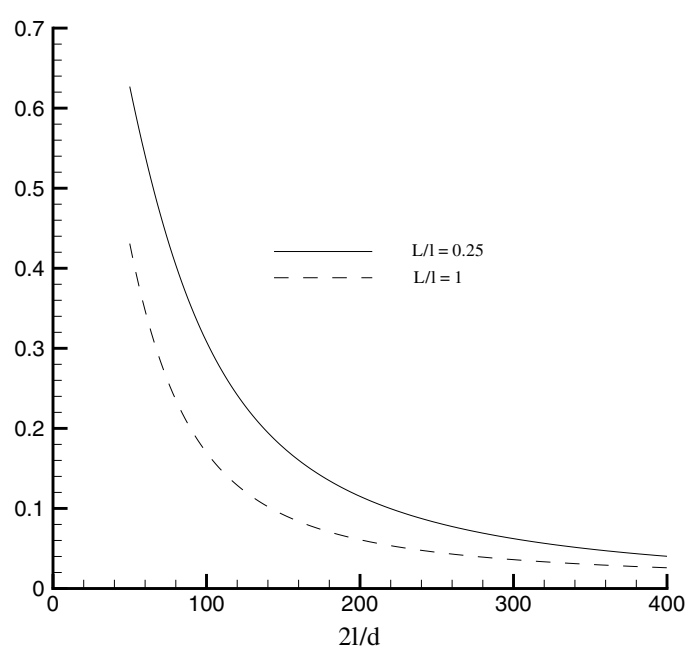

Figure 4. The heat transfer ratio $\sigma$ at different $2 l / d$ for $L / l=0.25$ and 1.0 .

and the convection from the wire, $\psi$, is given by

$$
\psi=\frac{\pi l k_{f} N u_{w} a}{\alpha} .
$$

$\sigma$ decreases when either $2 l / d$ or $L / l$ increases. At $2 l / d=$ $50, \sigma=43 \%$ and $63 \%$ for, respectively, $L / l=1.0$ and 0.25 . Champagne et al (1967) measured relative conduction end losses using temperature measurements and it was found that, for wires with $2 l / d \approx 200$ with short stubs, $\sigma \approx 7 \%$. Figure 4 shows that, for $\sigma=7 \%, 2 l / d$ needs to be 260 for $L / l=0.25$, while for $L / l=1$, this can be achieved with $2 l / d=170$ only. This shows that specification of a minimum value of $2 l / d$ alone is not sufficient and that stub length also needs to be taken into account.

Estimates of the heat transfer ratio, $\sigma$, in table 2, corresponding to the temperature distributions of figures 2 and 3 , assume a stub that is very large so that $\theta_{s}$ may be taken as constant. These estimates for $L / l=0.5$ may be compared with the data of figure 4 in which $L / l=0.25$ and 1.0. Model estimates of $\sigma$ for $2 l / d=200$ and 400 are slightly low because Freymuth's conduction-only model underestimates $\mathrm{d} \theta / \mathrm{d} \chi$ close to the wire-stub junction and therefore underestimates $K$ in equation (2.12) also. At smaller $2 l / d$, the model can be expected to be better. However, at small $\sqrt{c_{0}} l$ when conduction end-losses are larger, the model becomes increasingly sensitive to the assumption that $\theta_{s}=\theta_{x}(1)$ when it would be more appropriate to take a weighted mean stub temperature. At $2 l / d=100$, the model slightly overestimates $K$, whereas, at $2 l / d=50$, the opposite occurs. More importantly however, these results confirm that, at $2 l / d=200, \sigma<7 \%$ if $\sqrt{c_{0}} l \geqslant 3.0$, consistent with the results of Champagne et al (1967). For calculations at higher Reynolds numbers therefore, we use $\sigma<7 \%$ as the criterion for a minimum acceptable $2 l / d$.

Figure 5 shows the variation of $2 l / d$ with stub length, $0.25<L / l<4.0$, for a $5 \mu \mathrm{m}$ Pt wire at $R e_{w}=10$ with the requirement that $\sigma=7 \%$. The curve therefore represents the locus of minimum values $2 l / d$. The figure shows that for $0.25<L / l<2,2 l / d$ varies rapidly. However, for $L / l \geqslant 2$, the diameter ratio approaches a plateau where $2 l / d \approx 115$. Both figures 4 and 5 show that, for wires of smaller diameter, 


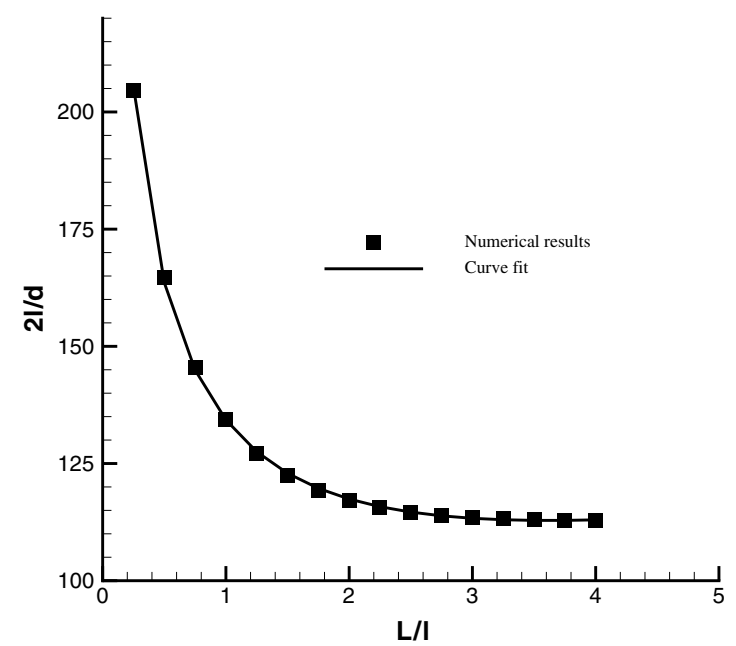

Figure 5. Variation of $2 l / d$ with $L / l$ for $R e_{w}=10$ and $\sigma=7 \%$ (Pt wire)

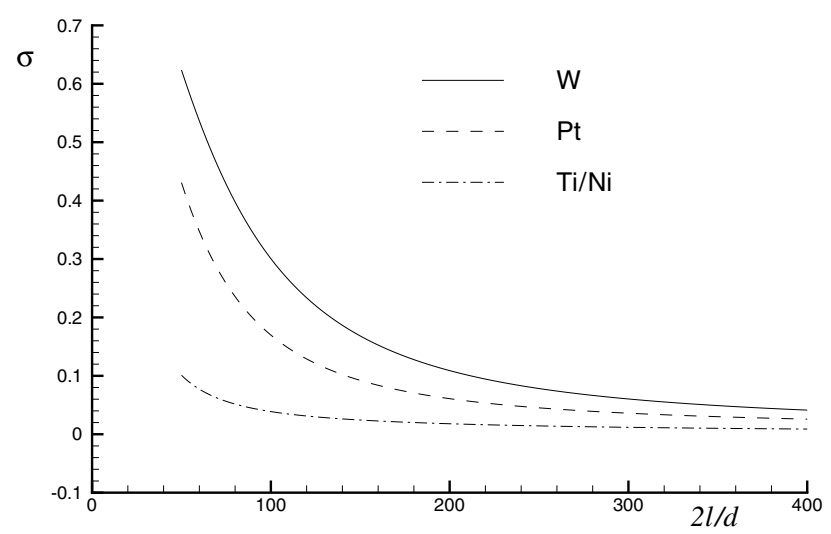

Figure 6. The heat transfer ratio $\sigma$ for different hot-wire materials; $R e_{w}=3.2, a=0.82, L / l=1.0, d=5 \mu \mathrm{m}$.

$L / l$ could be smaller. However, other factors then become important: a minimum stub length is helpful in avoiding the influence of vortex shedding from the prongs, but longer stub lengths lead to problems concerning the dynamic response caused by their increased resistance and temperature. These conflicting requirements are dealt with in section 4 .

\subsection{The effect of hot-wire material}

Figure 6 shows $\sigma$ plotted against $2 l / d$ for the three different hot-wire materials listed in table 1. These are platinum, tungsten and titanium/nickel (Ti/Ni: $55 \% \mathrm{Ti}$ and $45 \% \mathrm{Ni}$ ). The mechanical strengths of the three materials are about the same although the melting point temperatures are quite different. However, hot wires are normally operated at less than $300{ }^{\circ} \mathrm{C}$. There are significant differences between the values of $k_{w}$, with the low value for $\mathrm{Ti} / \mathrm{Ni}$ making it attractive for minimizing end-conduction effects. Figure 6 clearly shows that the thermal conductivity of the wire material has a strong effect on $\sigma$. For tungsten wire, $2 l / d$ needs to be 270 for $\sigma=7 \%$, for platinum wire, 170 and for $\mathrm{Ti} / \mathrm{Ni}$ wire, only 64. The normally used length-to-diameter ratio of about 200 is clearly an estimate based on Pt wire. This comparison also

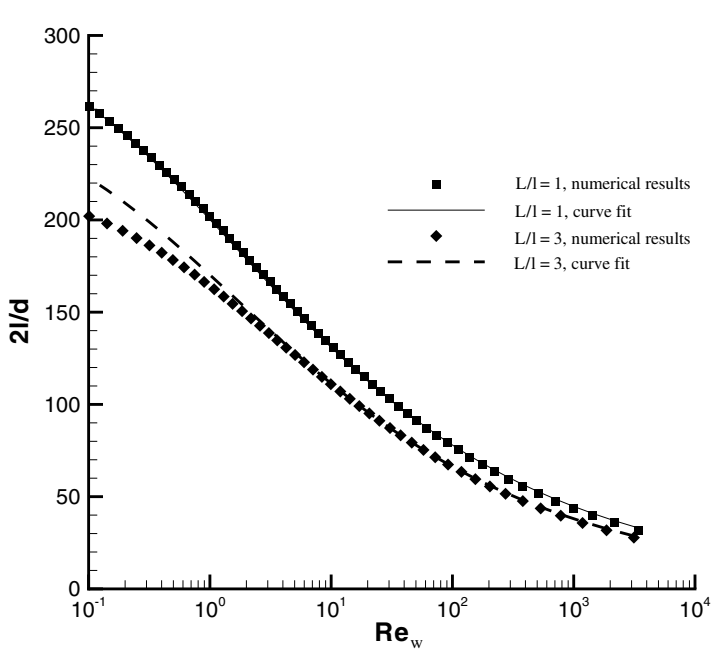

Figure 7. $2 l / d$ for $\sigma=7 \%$ as a function of Reynolds number for $L / l=1.0$ and 3.0. $5 \mu \mathrm{m}$ diameter Pt wire. Numerical results and curve fits, equations (2.14) and (2.15).

shows that $\mathrm{Ti} / \mathrm{Ni}$ would make an excellent material for hot wires.

\subsection{The effect of Reynolds number}

So far, the simulations have been undertaken at $R e_{w}=3.2$ and 10 . We now assess the minimum permissible values of $2 l / d$ for $\sigma=7 \%$ in the range $0.1 \leqslant R e_{w} \leqslant 3300$. Figure 7 shows their locus for a $5 \mu \mathrm{m} \mathrm{Pt}$ wire for $L / l=1$ and 3 . As expected, the required $2 l / d$ decreases with increasing Reynolds number. For $L / l=1$ at $R e_{w}=1,2 l / d=216$, while at $R e_{w}=100,2 l / d$ will only need to be 82 for the maximum acceptable value of $\sigma=7 \%$. For the present application to turbulent pipe flow at high Reynolds numbers, $R e_{D}$ (based on pipe diameter) up to $5.7 \times 10^{6}$, the equivalent wire Reynolds number, $R e_{w} \approx 250$ (see table 3 ). According to figure $7,2 l / d$ can be as low as 60 for $L / l=1$. When $L / l$ is increased to $3,2 l / d$ may be reduced even further to 50 . This represents a three- or four-fold improvement in spatial resolution over the accepted criterion $2 l / d \approx 200$ appropriate for atmospheric conditions. Moreover, shorter wires have shorter thermal time constants, suggesting that the frequency response can also be improved. Note that use of $\mathrm{Ti} / \mathrm{Ni}$ as the hot-wire material would reduce $2 l / d$ even further to as low as about 20 .

In order to establish a guideline for a minimum acceptable length-to-diameter ratio, the criterion, $\sigma \leqslant 7 \%$, is used as an acceptable upper limit to end-cooling effects. Data from the calculations, principally, those of figure 5 for the effects of $L / l$ on $2 l / d$, and those of figure 7 for the effects of $R e_{w}$, are used to provide the following correlations:

$$
\begin{aligned}
\frac{2 l}{d}= & 4.13\left(1.0-0.248 \ln \frac{L}{l}+0.0963\left[\ln \frac{L}{l}\right]^{2}\right) \\
& \times\left(\frac{k_{w}}{k_{f} N u_{w}}\right)^{0.48}
\end{aligned}
$$

for $0.25 \leqslant \frac{L}{l}<3.62$ and, for $\frac{L}{l} \geqslant 3.62$,

$$
\frac{2 l}{d}=3.41\left(\frac{k_{w}}{k_{f} N u_{w}}\right)^{0.48},
$$


The response of hot wires at high Reynolds numbers

Table 3. Principal parameters of data sets. $3 \mathrm{M} 8$ and $6 \mathrm{M} 9$ data sets were obtained using $2.5 \mu \mathrm{m}$ diameter $\mathrm{Pt} / \mathrm{Rh}$ wire. $3 \mathrm{M} 16$ data set was obtained using $2.5 \mu \mathrm{m}$ diameter $\mathrm{W}$ wire. $6 \mathrm{M} 10$ data set was obtained using $5 \mu \mathrm{m}$ diameter $\mathrm{Pt} / \mathrm{Rh}$ wire.

\begin{tabular}{llllcllllll}
\hline $\begin{array}{l}\text { Data } \\
\text { set }\end{array}$ & Anemometer & $R e_{D}$ & $R e_{w}$ & $\begin{array}{l}U_{c l} \\
\left(\mathrm{~m} \mathrm{~s}^{-1}\right)\end{array}$ & $2 l / d$ & $L / l$ & $\left.k_{1} \eta\right|_{\max }$ & $2 l / \eta$ & $\begin{array}{l}f_{c} \\
(\mathrm{kHz})\end{array}$ & $\begin{array}{l}f_{s} \\
(\mathrm{kHz})\end{array}$ \\
\hline 3M16 & $55 \mathrm{M} 12$ & $3.1 \times 10^{6}$ & 69.8 & 15.7 & 100 & 12 & 0.040 & 158 & 60 & 120 \\
3M8 & $55 \mathrm{M} 12$ & $3.1 \times 10^{6}$ & 68.4 & 8.00 & 100 & 19 & 0.049 & 127 & 75 & 75 \\
6M9 & $55 \mathrm{M} 12$ & $5.7 \times 10^{6}$ & 134 & 9.01 & 100 & 19 & 0.031 & 200 & 75 & 75 \\
6M10 & $90 \mathrm{C} 10$ & $5.5 \times 10^{6}$ & 287 & 9.69 & 120 & 1.0 & 0.012 & 531 & 100 & 75 \\
\hline
\end{tabular}

where $N u_{w}$ may be estimated using equation (1.1). Also shown in figure 7 are the curve fits using equations (2.14) and (2.15). They agree well with the calculation except for $L / l=3$ and $R e_{w}<1$. However, at such low Reynolds numbers, other effects such as buoyant convection not included in this analysis become significant.

\section{Experimental results}

\subsection{Experimental techniques}

Measurements have been made of the streamwise velocity component with hot wires with $2 l / d \approx 100$ at wire Reynolds numbers, $R e_{w}$, up to about 250 . The wires are made using standard etching techniques, using $\mathrm{Pt} / \mathrm{Rh}$ (Wollaston) and tungsten wire. Hot-wire calibration is performed using a fourth-order polynomial, the signal being sampled using 12bit A-D conversion. Three types of anemometer bridge are used: the 55M01 series DISA anemometer with both a standard 55M10 bridge (ratio 1:20) and a symmetrical 55M12 bridge (ratio 1:1), and the newer Dantec 'Streamline' system, 90C10 anemometer (standard bridge). Spectra are calculated using data records of $1800 \mathrm{~s}$ duration, except for the 3M16 data set. In this case, the data record length is 47 s only. Table 3 shows the principal parameters governing the flow conditions as well as details concerning the spatial and temporal resolution of the data. The spatial resolution is expressed non-dimensionally using the Kolmogorov length scale, $\eta$, as deduced from dissipation spectra assuming local isotropy. These estimates are typically $10 \%$ larger than those deduced from the third-order structure function. Here, $\left.k_{1} \eta\right|_{\max }$ is the non-dimensional maximum wavenumber resolution, where $\left.k_{1}\right|_{\max }=\pi / l$. The sampling frequency is such that the Nyquist frequency expressed as a wavenumber exceeds $\left.k_{1}\right|_{\max }$ for the worst case situation of data at the pipe centre line. The signal was low-pass filtered at the Nyquist frequency and standard FFT algorithms (Hanning window) are used to calculate the spectra as a function of streamwise wavenumber, $k_{1}=2 \pi f / U$.

The two data sets at $R e_{w} \approx 70$, identified as 3M16 and $3 \mathrm{M} 8$, differ principally in the mean velocity. Less significant are the differences in hot-wire material, $L / l$, frequency response, $f_{c}$, and sample frequency, $f_{s}$, which are due simply to the choices made by individual workers. The stub-wire length ratios are somewhat larger than those of the calculations in section 2. However, figure 5 shows that the effects of increasing $L / l$ are minimal for $L / l>3$.

\subsection{Spatial and temporal resolution}

The inherent problem of the $f^{2}$-ramp is illustrated by examining 'noise-floor' spectra obtained in air at nominally

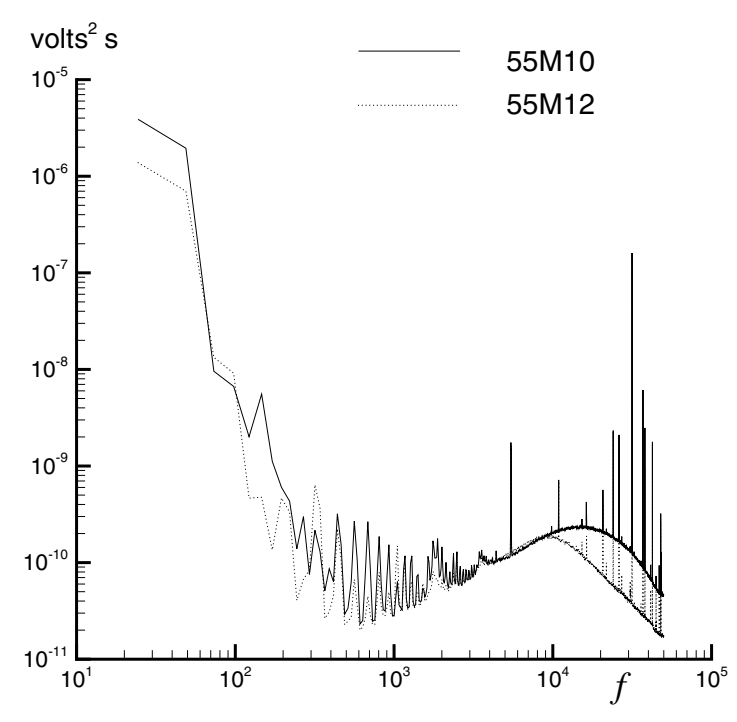

Figure 8. 'Noise-floor' spectra at nominally zero air speed: comparison of standard (55M10) and symmetrical (55M12) bridges.

zero velocity. Figure 8 compares frequency spectra for the standard (55M10) and symmetrical (55M12) bridges with an overheat ratio, $a \approx 0.7$. Both bridges show a noise ramp beginning at $f \approx 2 \mathrm{kHz}$. With the ordinate in units of (volts) $)^{2}$, the slope is close to $f^{1 / 2}$. Assuming a King's-law relationship between anemometer output and cooling velocity, this is equivalent to an $f^{2}$-spectral slope for an ordinate in units of (velocity) ${ }^{2} \mathrm{~s}$, and to a velocity amplitude proportional to $f$. This behaviour is very similar to the spectra in Saddoughi and Veeravalli (1996) obtained using a Dantec 56C17 bridge although the precise slope depends on the details of the amplifier design and bridge frequency response. As expected, the symmetrical bridge exhibits less noise at frequencies in excess of $10 \mathrm{kHz}$.

The effect of the $f^{2}$-ramp on data obtained at high Reynolds number and the potentially disastrous effect it can have on the interpretation of spectra is best illustrated by inspection of dissipation spectra obtained at the pipe centre line, $R e_{D}=3.1 \times 10^{6}$. The form of these spectra is given by $\epsilon_{\text {iso }}=15 v \int_{0}^{\infty} k_{1}^{2} \phi_{11}\left(k_{1}\right) \mathrm{d} k_{1}=15 v \int_{0}^{\infty} k_{1}^{2} \phi_{11}\left(k_{1} \eta\right) \mathrm{d} k_{1} \eta$,

where $\epsilon_{\text {iso }}$ is the isotropic estimate of the dissipation rate. Considerations of the energy budget show that, at the pipe centre line, equation (3.1) is a close approximation to the total dissipation rate, $\epsilon$. Poor resolution of the data either temporally or spatially would be evident if the dissipation spectra were not to show an appreciable maximum in the region 


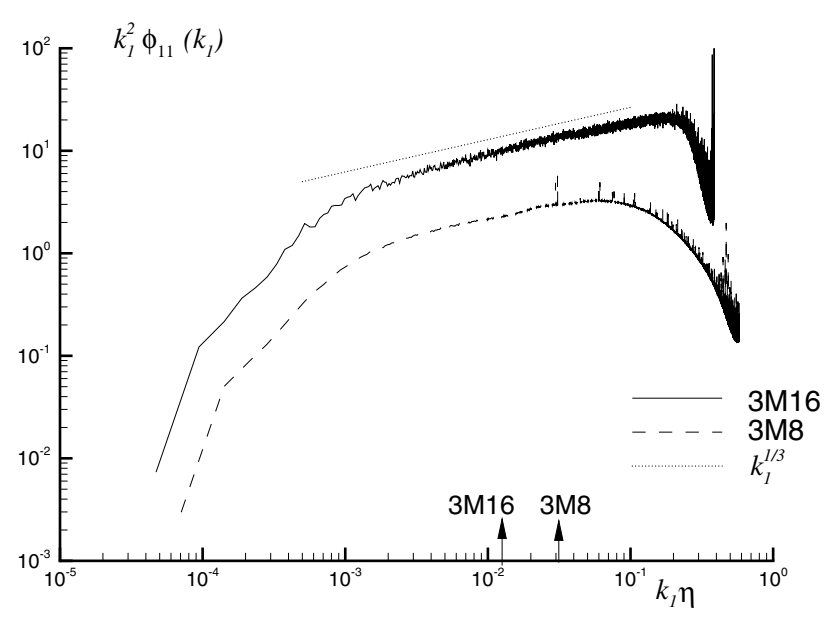

Figure 9. Dissipation spectra, $k_{1}^{2} \phi\left(k_{1}\right), \operatorname{Re}_{D}=3.1 \times 10^{6}$. Vertical arrows denote ordinate values corresponding to $f=2 \mathrm{kHz}$.

of $k_{1} \eta=0.25$ with, of course, a region of $1 / 3$ slope at slightly lower wavenumbers.

Figure 9 shows two spectra at the pipe centre line, using the data sets, one at high velocity (3M16), the other at lower velocity $(3 \mathrm{M} 8)$. The spectra are both obtained using the $55 \mathrm{M} 12$ symmetrical bridge, but at mean velocities that differ by a factor of about two while the Reynolds number is fixed at $R e_{D}=3.1 \times 10^{6}$. Both spectra are fully resolved in time, and the limit to the spatial resolution is similar in each case. However, using equation (3.1), $\epsilon_{\text {iso }}=2.8 \mathrm{~m}^{2} \mathrm{~s}^{-1}$ and $0.17 \mathrm{~m}^{2} \mathrm{~s}^{-1}$ for the high- and low-velocity data, respectively. Differences in anemometer frequency response are clear, despite the use of the same overheat ratio in each case, and the same amplifier gain. The frequency response at the lower velocity rolls off slightly more slowly than that at the higher velocity because of a slightly better filter setting. However, the most significant difference is the extended $1 / 3$ range at the higher velocity which is responsible for the much higher estimate of $\epsilon_{\text {iso }}$. This must be spurious and can reasonably be attributed to an $f^{2}$-ramp in the anemometer frequency response. Closer inspection of the spectrum at the lower velocity (3M8) suggests that it has a maximum at $k_{1} \eta \approx 0.06$ while that at the higher velocity peaks (spuriously) at $k_{1} \eta \approx 0.21$, which, on the face of it, is an acceptable value. Similarly, the anemometer response at the lower velocity is also inadequate for dissipation estimates: in this case, the high-wavenumber attenuation is the genuine consequence of poor spatial resolution which is masked by the $f^{2}$-ramp in the high-velocity data (3M16).

In figure 10 , the same data are compared using inertial subrange scaling:

$$
\frac{\phi_{11}\left(k_{1}\right)}{k_{1}^{-5 / 3} \epsilon^{2 / 3}}=C_{1} .
$$

Here, use of a linear ordinate makes the spectra sensitive to any anomalies in anemometer behaviour, and in particular, the spectral plateau at high Reynolds numbers should asymptote to the constant, $C_{1} \approx 0.5$. The ordinate value for the lowvelocity data is clearly in error and is due to the poor spatial resolution that leads to an underestimate in $\epsilon_{\text {iso }}$. For the highvelocity data, the poor spatial resolution is compensated by

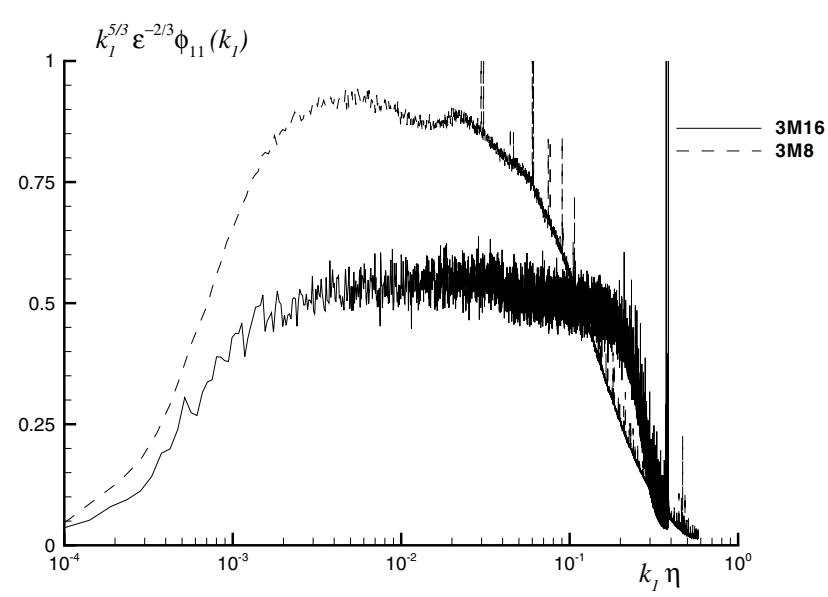

Figure 10. Spectra with inertial-subrange scaling: $k_{1}^{5 / 3} \epsilon^{-2 / 3} \phi_{11}\left(k_{1}\right)$.

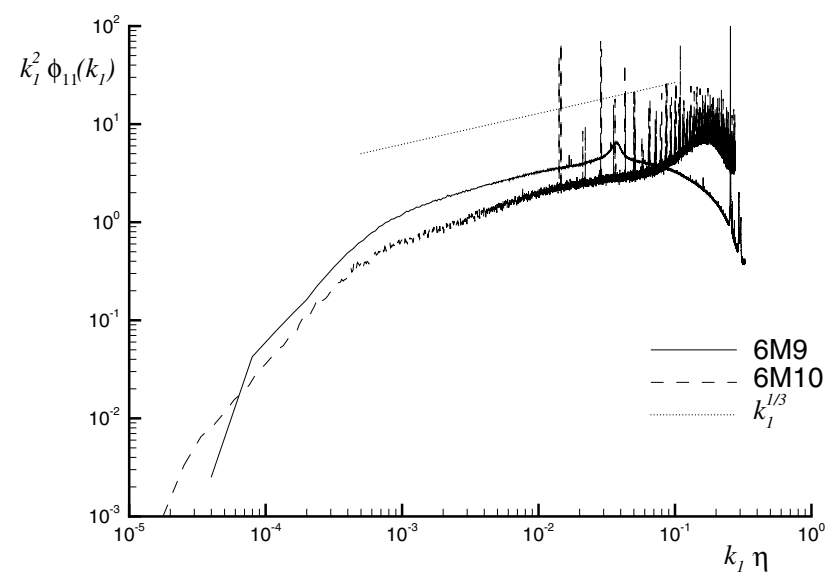

Figure 11. Dissipation spectra, $k_{1}^{2} \phi\left(k_{1}\right), R e_{D}=5.7 \times 10^{6}$.

the $f^{2}$-ramp in the frequency response: taking $\phi_{11} \sim f^{2}$, $\phi_{11} k_{1}^{-5 / 3} \epsilon^{2 / 3} \sim f^{1 / 3}$ so that the ordinate is more or less constant with frequency. Therefore, the estimate of $\epsilon_{\text {iso }}$ is approximately correct, but for the wrong reasons. It might also appear that these spectra behave in a similar fashion to those reported in Saddoughi and Veeravalli (1994), where similarly scaled spectra of the streamwise velocity component in a highReynolds-number boundary layer also show bumps. However, as we shall show, the effect in this case appears to be directly related to the appearance of heat waves along the hot wire. In summary, the expectation based on the evidence of figures 9 and 10, that the high-velocity data set (3M16) is an acceptable one, is entirely false.

\subsection{Dynamic conduction end-losses}

Figure 11 shows spectra obtained on the pipe centre line at $R e_{D}=5.7 \times 10^{6}$ using the $55 \mathrm{M} 12$ symmetrical bridge (6M9), and at $R e_{D}=5.5 \times 10^{6}$ with the $90 \mathrm{C} 10$ standard bridge (6M10). The spectra are plotted in dissipation form, equation (3.1). In the case of the 55M12 bridge, there is a clear bump on the spectrum at $k_{1} \eta \approx 0.035$. This effect is most prevalent for spectra on the pipe centre line where the signal-to-noise ratio is lowest. In fact, only for spectra taken at distances from the centre line greater than 
$70 \%$ of the pipe radius is the signal sufficiently large for the effect to be masked. Using equation (1.5) to estimate a circular frequency above which heat waves are attenuated gives $\omega_{l} \approx 8.3 \times 10^{3}$ for $\mathrm{Pt} / \mathrm{Rh}$ wire $0.25 \mathrm{~mm}$ long and with diffusivity, $\wp \approx 1.3 \times 10^{-5} \mathrm{~m}^{2} \mathrm{~s}^{-1}$. This corresponds to $\left.k_{1} \eta\right|_{l} \approx 0.012$. The equivalent spectrum obtained using the $90 \mathrm{C} 10$ standard bridge (6M10) has a pronounced bump at $k_{1} \eta \approx 0.17$. However, the corresponding estimate using equation (1.5) is $\left.k_{1} \eta\right|_{l} \approx 0.0017$ only, owing to the increased wire length. Clearly, the bump at $k_{1} \eta \approx 0.17$ is more likely to be caused by the anemometer frequency response. Differences between the estimated and measured values of $\left.k_{1} \eta\right|_{l}$ for the 6M9 data set could be attributed to the influence of the long stubs $(L / l=19)$ which, while making the wire temperature more uniform, do increase the temperature of the stub and so increase generation within it and convection from it. Under these circumstances, it is unlikely that equation (1.5) will be accurate. The effect of long stubs is to introduce an additional time constant with a value that is several orders of magnitude larger than that for heat waves in the wire.

Table 2 also shows estimates of $\sigma^{\prime}$ given by equation (1.6) and calculated using Freymuth's solution with no small overheat assumptions. Again, we assume that $\theta_{s}=\theta_{x}(1)$ using the solutions of figure 2 : therefore, as in the estimate of $\sigma$, the value of $\sigma^{\prime}$ is appropriate for a hot wire with a stub length $L / l=0.5$. The estimates show, for a wire at atmospheric conditions with $2 l / d \approx 200$ and $L / l=0.5$, that $\sigma^{\prime} \approx 2 \%$. Using a time-dependent thermal model of a hot wire with a modelled ideal feedback amplifier, Morris and Foss (2003) have calculated the transient response of a tungsten hot wire with $2 l / d=200$ and $L / l=0.1$ under similar conditions. Their calculations suggest that $\sigma^{\prime}=7 \%$ in agreement with Freymuth's conduction-only solution. This larger estimate is the result of the shorter stub length.

Our 'benchmark' values of end-conduction are given by $\sigma<7 \%$ and $\sigma^{\prime}<2 \%$ for a hot wire with $2 l / d=200$ at $R e_{w} \approx 10$. The static calculations of figure 7 show that the minimum value of $2 l / d$ for $\sigma<7 \%$ when $L / l \approx 1.0$ at $R e_{w}=287$ (data set $6 \mathrm{M} 10$ ) is about 60 only. The data sets used for subsequent analysis, 3M8 and 6M9, used wires for which $2 l / d=100$ and $L / l=19$ (Morrison et al 2004). Using the calculated temperature distributions for a Pt wire at $R e_{w}=250$ with $2 l / d=100$ and $a=0.82$ together with Freymuth's conduction-only model, yield estimates, $\sigma=2.3 \%$ and $\sigma^{\prime}=0.84 \%$. Therefore, end-conduction effects for the $3 \mathrm{M} 8$ and $6 \mathrm{M} 9$ data sets are significantly less than those associated with more conventional measurements at $R e_{w} \approx 10$ with $2 l / d \approx 200$.

\section{Conclusions and recommendations}

Numerical solutions of the steady-state heat-balance equation for a hot wire and its cylindrical support stub show that the temperature distribution depends on the length-to-diameter ratio, the stub length, the material used for the hot wire and the Reynolds number. Simple assumptions such as that of a linear temperature distribution in the stub are not accurate, especially at large $2 l / d$ when the wire temperature distribution becomes more uniform but the temperature distribution near the wirestub junction becomes more complicated. By specifying a maximum acceptable fraction of heat loss by conduction to the stubs, $\sigma=7 \%$, the curve fits to the numerical solutions given by equations (2.14) and (2.15) may be used to calculate a minimum $2 l / d$ for a given Reynolds number, stub length and wire material. It is shown that the accepted minimum for $2 l / d$ of 200 refers only to the use of platinum wire at typical velocities at atmospheric conditions. For tungsten wire, this ratio should be increased to about 270. A better hot-wire material is found to be titanium/nickel which has similar mechanical and electrical properties as those of platinum, but has a lower thermal conductivity. Under atmospheric conditions with a cooling velocity of about $10 \mathrm{~m} \mathrm{~s}^{-1}, 2 l / d$ may be as low as 60 and still meet the requirement, $\sigma \leqslant 7 \%$.

At Reynolds numbers $R e_{w}>100$, further reductions in $2 l / d$ are possible without compromising the limit on $\sigma$. Similarly, wire lengths can be reduced merely by reducing its diameter. However, practical considerations suggest a minimum diameter of $2.5 \mu \mathrm{m}$. At high wire Reynolds numbers, an optimal anemometer frequency response is both more important yet more difficult to achieve. The inevitable appearance of an $f^{2}$-ramp in velocity spectra measured with analogue anemometers means that the frequency response can appear to be artificially good. Even with a carefully tuned bridge, the effect of heat waves is likely to become more apparent at high $R e_{w}$ as the bridge gain is reduced to maintain stability. As the wire length increases (or equivalently, as $R e_{w}$ increases for a wire of fixed length), the temperature distribution in the vicinity of the wire-stub junction becomes complicated by the effects of heat generation within the stub and convection from it. Under these circumstances, the effect of heat waves as the solution of the one-dimensional heat conduction equation is difficult to discuss sensibly. Even so, equation (1.5) provides an acceptably accurate estimate of the maximum frequency up to which these waves are likely to be a problem. It suggests that their effect can be minimized by: (a) using wire material of low thermal conductivity, and (b) increasing the wire length. Thus, replacing platinum wire with a titanium/nickel one provides a seven-fold reduction in $\omega_{l}$. Unfortunately, (b) is counter-productive in terms of resolution.

\section{Acknowledgments}

The support of ONR under grant nos N00014-98-1-0525, N00014-99-1-0340 and N00014-03-1-0320 is gratefully acknowledged. JFM is indebted to the Engineering and Physical Sciences Research Council (grants GR/M64536/01 and GR/R48193/01), the Royal Academy of Engineering (England), and the Leverhulme Trust (grant F/07058/H) for financial support.

\section{References}

Brunn H H 1995 Hot-Wire Anemometry: Principles and Signal Analysis (Oxford: Oxford University Press)

Champagne F H, Sleicher C A and Wehrmann O H 1967 Turbulence measurements with inclined hot wires: Part 1. Heat transfer experiments with inclined hot wire J. Fluid Mech. 28 153-75

Collis D C and Williams M J 1959 Two-dimensional convection from heated wires at low Reynolds numbers J. Fluid Mech. 6 357-84

Corrsin S 1963 Turbulence: experimental methods Handbuch der Physik vol 8 (Berlin: Springer) pp 524-90 
Fand R M and Keswani K K 1972 A continuous correlation equation for heat transfer from cylinders to air in cross-flow for Reynolds numbers from $10^{-2}$ to $2 \times 10^{5}$ Int. J. Heat Mass Transfer 15 559-62

Fingerson L M and Freymuth P 1983 Thermal anemometers Fluid Mechanics Measurements ed R J Goldstein (New York, NY: Hemisphere Publishing) pp 99-154

Freymuth P 1968 Noise in hot-wire anemometers Rev. Sci. Instrum. 39 550-7

Freymuth P 1977 Frequency response and electronic testing for constant temperature hot-wire anemometers J. Phys. E: Sci. Instrum. 10 705-10

Freymuth P 1979 Engineering estimation of heat conduction loss in constant temperature thermal sensors TSI Quart. 5 3-8

Freymuth P and Fingerson L M 1997 Hot-wire anemometry at very high frequencies: effect of electronic noise Meas. Sci. Technol. 8 115-6

Hinze J O 1975 Turbulence 2nd edn (New York: McGraw-Hill)

Li J 2001 An investigation on the length to diameter ratio of hot wire filament in turbulence measurements Proc. 14th Australasian Fluid Mechanics Conf. (Adelaide)

McKeon B J, Li J, Jiang W, Morrison J F and Smits A J 2004 Further observations on the mean velocity in fully-developed pipe flow J. Fluid Mech. 501 135-47
Morris S C and Foss J F 2003 Transient thermal response of a hot-wire anemometer Meas. Sci. Technol. 14 251-9

Morrison J F, Jiang W, McKeon B J and Smits A J 2002a Reynolds-number dependence of streamwise velocity fluctuations in turbulent pipe flow AIAA 2002-0574

Morrison J F, Jiang W, McKeon B J and Smits A J 2002b Reynolds-number dependence of streamwise velocity spectra in turbulent pipe flow Phys. Rev. Lett. 88214501

Morrison J F, Jiang W, McKeon B J and Smits A J 2004 Scaling of the streamwise velocity component in turbulent pipe flow J. Fluid Mech. at press

Perry A E 1982 Hot-wire Anemometry (Oxford: Clarendon)

Perry A E, Smits A J and Chong M S 1979 The effects of certain low frequency phenomena on the calibration of hot wires J. Fluid Mech. 90 415-31

Saddoughi S G and Veeravalli S V 1994 Local isotropy in turbulent boundary layers at high Reynolds numbers J. Fluid Mech. $268333-72$

Saddoughi S G and Veeravalli S V 1996 Hot-wire anemometry at very high frequencies Meas. Sci. Technol. 7 1297-300

Zagarola M V 1996 Mean flow scaling in turbulent pipe flow $P h D$ Thesis Princeton University

Zagarola M V and Smits A J 1998 Mean-flow scaling of turbulent pipe flow J. Fluid Mech. 373 33-79 